\title{
Ether lipid 1-O-octadecyl-2- $O$-methyl-3-glycero-phosphocholine inhibits cell-cell adhesion through translocation and clustering of E-cadherin and episialin in membrane microdomains
}

\author{
SEVERINE VAN SLAMBROUCK ${ }^{1}$, JOHN HILKENS ${ }^{2}$ and WIM F.A. STEELANT ${ }^{1}$ \\ ${ }^{1}$ Laboratory of Biochemical and Biomedical Research, Department of Chemistry, New Mexico Tech, 801 Leroy Place, \\ Socorro, NM 87801, USA; ${ }^{2}$ Department of Molecular Genetics, The Netherlands Cancer Institute, \\ Plesmanlaan 121, 1066 CX Amsterdam, The Netherlands
}

Received July 30, 2007; Accepted September 11, 2007

\begin{abstract}
The ether lipid 1-O-octadecyl-2- $O$-methyl-3glycero-phosphocholine (ET-18-OMe) inhibits cell-cell adhesion and induces invasiveness of breast cancer cells. Previously, we showed that a loss of cell-cell adhesion was due to sterical hindrance of E-cadherin by the anti-adhesive properties of the cell surface mucin episialin. Here, we demonstrated that the ether lipid ET-18-OMe induced the translocation of E-cadherin and episialin to membrane microdomains, enriched in glycosphingolipids, known to be involved in cell-cell adhesion and cell signaling. In addition, it was found that E-cadherin and clusters of episialin colocalized and associated with the glycosphingolipid, MSGb5, upon treatment with ET-18-OMe. Together, these results suggest that ET-18-OMe inhibits cell-cell adhesion by inducing the translocation of E-cadherin and episialin into MSGb5-enriched membrane microdomains, which leads to clustering and colocalization of the pro-adhesive E-cadherin and the antiadhesive episialin thereby inhibiting cell-cell adhesion.
\end{abstract}

\section{Introduction}

The plasma membrane contains specialized membrane microdomains, enriched in glycosphingolipids (GSLs) (1). Several studies suggested that these membrane microdomains

Correspondence to: Dr Wim F.A. Steelant, Laboratory of Biochemical and Biomedical Research, Department of Chemistry, New Mexico Tech, 801 Leroy Place, Socorro, NM 87801, USA

E-mail: steelant@nmt.edu

Abbreviations: GSL, glycosphingolipid; SDS-PAGE, sodium dodecyl sulfate polyacrylamide gel electrophoresis; ECL, enhanced chemiluminescence; TR, texas-red; FITC, fluorescein isothiocyanate; PBS, phosphate-buffered saline

Key words: glycosphingolipid, microdomain, cell-cell adhesion, E-cadherin, episialin, ether lipid 1-O-octadecyl-2-O-methyl-3glycero-phosphocholine play fundamental roles in numerous cellular functions by promoting segregated compartmentalization of membrane proteins and lipids (2-4). In tumor cells, GSLs were identified as tumor-associated antigens (5) and function as mediators of cell adhesion and modulators of signal transduction, through differential organization, clustering and assembly with specific membrane proteins and signal transducers (4).

The ether lipid 1-O-octadecyl-2-O-methyl-3-glycerophosphocholine (ET-18-OMe) is a selective antitumor agent, known to inhibit cancer cell proliferation (6) and to induce apoptosis (7). The mechanism by which ET-18-OMe exerts the latter effect relates to its ability to interact with cell membranes, thereby reorganizing crucial molecules in membrane microdomains and interfering with subsequent signaling (8). Previously, we showed that ET-18-OMe induced a loss of cell-cell adhesion and stimulated the invasion of MCF-7 breast cancer cells into collagen type I, which resulted in the inhibition of E-cadherin-mediated adhesion by episialin. Both E-cadherin and episialin, membrane-associated glycoproteins, were found to have colocalized in MCF-7 cells upon ET-18-OMe treatment (9).

Given these previous observations, we investigated whether an ET-18-OMe-induced loss of cell-cell adhesion results from the reorganization and/or association of E-cadherin, episialin and glycosphingolipids in membrane microdomains.

\section{Materials and methods}

Cell culture. MCF-7/AZ and MCF-7/6 cells, variants of the human mammary carcinoma cell family MCF-7, were maintained on a tissue culture plastic substrate (Nunc, Rochester, NY) in a mixture of Dulbecco's modified Eagle's medium (DMEM) and HAM F12 (50/50) (Invitrogen, Carlsbad, CA) supplemented with $250 \mathrm{IU} / \mathrm{ml}$ penicillin, $100 \mathrm{mg} / \mathrm{ml}$ streptomycin and $10 \%$ fetal bovine serum (FBS) (Invitrogen), at $37^{\circ} \mathrm{C}$ in a humidified atmosphere containing $10 \% \mathrm{CO}_{2}$. MCF-7/AZ cells differ from MCF-7/6 cells in that they are not invasive when tested in precultured chick heart invasion in vitro (10).

Reagents. ET-18-OMe (clinical grade) was provided by Dr P. Hilgard (ASTA Medica, Frankfurt am Main, Germany). 
Cells were cultured in the presence of $15 \mu \mathrm{g}$ ET-18-OMe/ml culture medium for the indicated times. Drug toxicity was evaluated through the measurement of mitochondrial dehydrogenase activities with an MTT reagent (Sigma, St. Louis, MO) (11). Micro-BCA and BCA protein assay reagent kits were obtained from Pierce (Rockford, IL). The Vectastain ABC-AMP kit was from Vector Laboratories (Burlingame, CA).

Antibodies. The mouse anti-GSL antibodies used in this study were: IgM 1A4 (E10) to Gb3, IgM MK1-8 to GM2, IgM 9G7 to Gb4, IgM SSEA-3 to Gb5 (12) and IgM RM1 to MSGb5 (13). Mouse IgG3 to MSGb5, developed by Davor Solter, was obtained from the Development Studies Hybridoma Bank developed under the auspices of the NICHD and maintained by the University of Iowa, Department of Biological Sciences, Iowa City, IA. The mouse antibody directed to E-cadherin was from Takara Bio Inc. (Kyoto, Japan), while the rat antibody recognizing E-cadherin was from Abcam Inc. (Cambridge, MA). Mouse episialin antibodies were from Millipore (Temecula, CA) and rabbit anti-episialin was from Abcam Inc. Secondary biotinylated anti-rabbit and anti-mouse, FITClabeled anti-mouse, TR-labeled anti-rabbit and anti-rat secondary antibodies were from Vector Laboratories.

E-cadherin and episialin in membrane microdomains and soluble fractions. Cells treated for $24 \mathrm{~h}$ with ET-18-OMe were used for the preparation of postnuclear fractions in $1 \%$ Triton $\mathrm{X}-100$ followed by a sucrose gradient-density centrifugation to isolate 12 fractions, including membrane microdomains (fractions 4-6) and high-density soluble fractions (10-12) as described $(14,15)$. Aliquots, containing the same quantity of proteins were boiled for $5 \mathrm{~min}$ in an SDS-PAGE sample buffer, containing 5\% ß-mercaptoethanol, electrophoresed on $7.5 \%$ SDS-PAGE and transferred to PVDF membranes (Immobilon-P) (Bio-Rad Laboratories, Hercules, CA). After transfer, the membranes were incubated with the relevant antibodies, followed by incubation with a secondary biotinylated antibody (1:1000) and developed by the ECL (Vectastain ABC-AMP) detection kit. Membranes were imaged and analyzed on the BioChemi System and analysis software (UVP, Upland, CA).

Immunofluorescence detection of episialin, E-cadherin and GSLs. Cells, grown on cover glasses and placed in 24-well plates, were treated for $24 \mathrm{~h}$. Subsequently, the cover glasses with cells were removed, washed with ice-cold PBS and fixed with $3 \%$ paraformaldehyde. After washing, the fixed cells were incubated for $1 \mathrm{~h}$ with primary antibodies, against E-cadherin, episialin and glycosphingolipids, followed by incubation with secondary FITC- or TR-labeled antibodies. Stained cells were mounted with Glycergel mounting medium (Dako Corp., Carpinteria, CA) containing 1\% 1,4-diazabicyclo[2.2.2] octane. Confocal laser scanning was performed with a Leica DM IRBE fluorescence microscope and Leica TCS NT confocal unit.

Immunoprecipitation of MSGb5 and the association with E-cadherin and episialin. Confluent cultures (70\%) treated for the indicated times with ET-18-OMe were lysed in $0.5 \mathrm{ml}$ lysis buffer containing 1\% Triton X-100, 1\% NP-40 and the following inhibitors: aprotinin $(10 \mu \mathrm{g} / \mathrm{ml})$, leupeptin $(10 \mu \mathrm{g} /$ $\mathrm{ml}), \operatorname{PMSF}(1.72 \mathrm{mM}), \mathrm{NaF}(100 \mathrm{mM}), \mathrm{NaVO}_{3}(500 \mu \mathrm{M})$ and $\mathrm{Na}_{4} \mathrm{P}_{2} \mathrm{O}_{7}(500 \mu \mathrm{g} / \mathrm{ml})$. Lysates, containing $2000 \mu \mathrm{g}$ of protein, were mixed with protein G-Sepharose beads (Amersham Biosciences, NJ) (50 $\mu 1$, packed) and placed on a rotator for $30 \mathrm{~min}$ at $4^{\circ} \mathrm{C}$, to preclear non-specific binding. After centrifugation at $1,000 \mathrm{rpm}$, to collect supernatant, $2 \mu 1$ of IgG3 MSGb5 antibody was added and rotated at $4{ }^{\circ} \mathrm{C}$ overnight. Subsequently, protein G-Sepharose beads were added, rotated at $4^{\circ} \mathrm{C}$ for $2 \mathrm{~h}$ and centrifuged to collect the beads. The beads were washed with lysis buffer and immunoprecipitates were resolved in $100 \mu \mathrm{l}$ SDS-PAGE sample buffer and heated to $95^{\circ} \mathrm{C}$ for $5 \mathrm{~min}$. Supernatants were subjected to $7.5 \%$ SDSPAGE, transferred electrophoretically to PVDF membranes, incubated with the relevant antibodies and analyzed and imaged as described above.

Statistics. All treatments were matched and carried out at least 3 times. The Student's t-test $(95 \%, \mathrm{p}<0.05)$ was used for statistical evaluation. Levels were quantified using the statistical software Scion image (Scion Corporation, MD).

\section{Results}

E-cadherin and episialin in membrane microdomains of $M C F-7 / A Z$ vs. MCF-7/6 cells. E-cadherin and episialin were associated with membrane microdomains [low density (fr. 4-6) and soluble high density fractions (10-12)] in both MCF-7/AZ and MCF-7/6 cells. Upon ET-18-OMe treatment, the levels of E-cadherin and episialin increased in fr. 4-6 and simultaneously decreased in fr. 10-12 in the MCF-7/AZ cells (Fig. 1, left panel), while no altered expression levels were observed in the MCF-7/6 cells (Fig. 1, right panel).

Localization and distribution patterns of E-cadherin and episialin and various GSLs in MCF-7/AZ vs. MCF-7/6 cells. Localization and distribution patterns of E-cadherin and episialin and diverse GSLs in the membrane microdomains of MCF-7/AZ and MCF-7/6 cells were observed by confocal microscopy. The GSL composition of the two cell lines was previously determined by high-performance thin-layer chromatography $(16,17)$. Fig. 2A shows the presence of E-cadherin at the lateral surface, whereas episialin was exclusively present at the apical surface in the two cell lines. Incubation of the MCF-7/AZ cells with ET-18-OMe led to the clustering and translocation of episialin to the lateral surface and colocalization with E-cadherin, in line with our previous study (9) (left panel), while no such effect was observed in the MCF-7/6 cells (Fig. 2A, right panel). An analysis of the localization of GSLs, upon ET-18-OMe treatment, revealed no major changes (data not shown) in the two cell lines except for MSGb5 in the MCF-7/AZ cells, which clustered and translocated to the lateral surface. In addition, upon treatment, both E-cadherin and episialin were found to have colocalized with MSGb5 patches in the MCF-7/AZ cells (Fig. 2B and C, left panel) but not in the MCF-7/6 cells (Fig. 2B and C, right panel).

Association of MSGb5 with E-cadherin and episialin. The association MSGb5 with E-cadherin and episialin was shown 

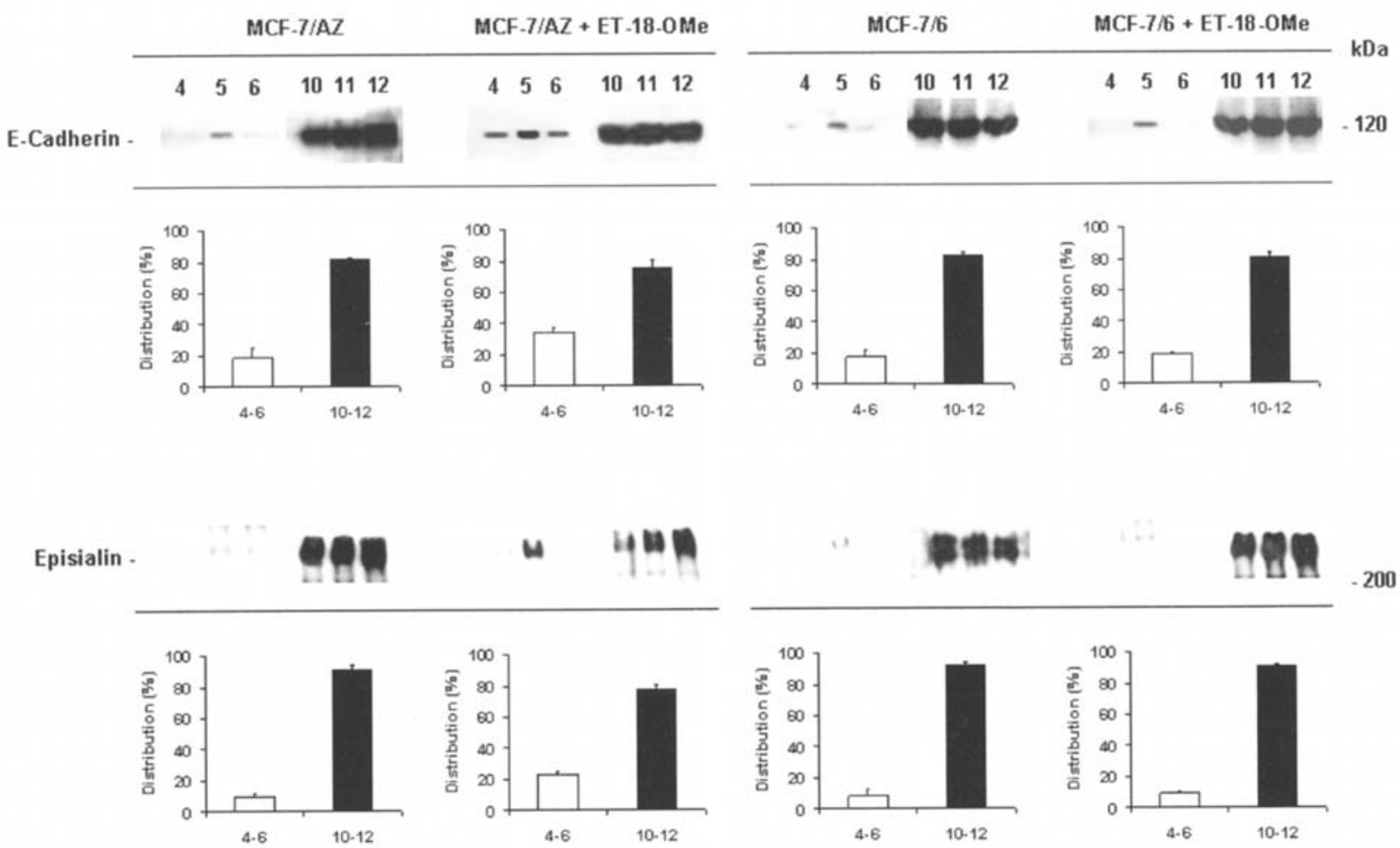

Figure 1. Expression levels of E-cadherin and episialin in membrane microdomains (fr. 4-6) and (10-12) of MCF-7/AZ and MCF-7/6 cells. MCF-7/AZ and MCF-7/6 cells were treated for $24 \mathrm{~h}$ with ET-18-OMe. Membrane microdomains were isolated by sucrose gradient centrifugation and each fraction was analyzed by SDS-PAGE and immunoblotted with antibodies against E-cadherin (upper panel) and episialin (lower panel), accompanied by a Scion image densitometry analysis of the bands of E-cadherin and episialin in membrane microdomains (white columns) and in fr. 10-12 (black columns). Bars indicate standard deviation.

by immunoprecipitation experiments. Both E-cadherin and episialin were detected in MSGb5 immunoprecipitates in MCF-7/AZ and MCF-7/6 cells. After treatment with ET-18OMe increasing E-cadherin and episialin levels were found to be associated with MSGb5 in MCF-7/AZ cells (Fig. 3A and B, left panel), while no differences in levels could be observed in MCF-7/6 cells (Fig. 3A and B, right panel).

\section{Discussion}

The ether lipid, 1-O-octadecyl-2- $O$-methyl-3-glycerophosphocholine, is a synthetic analogue of 2-lysophosphatidylcholine and considered the prototype of the antitumor lipid class. Several studies revealed that the anticancer activity of ET-18-OMe is caused by the induction of apoptosis $(7,18)$, through interaction with cell membranes $(8,19)$. However, we found in a previous study that ET-18-OMe treatment induced a loss of E-cadherin-mediated cell-cell adhesion in MCF-7/AZ breast cancer cells (9), representing a critical step in tumor cell invasion and metastasis (20). Notably, the loss of cell-cell adhesion was not detected in the MCF-7/6 breast cancer cells and enabled us to identify differences responsible for the ET18-OMe-induced effect. We showed that ET-18-OMe did not affect the cadherin-catenin complex but induced the loss of cell-cell adhesion due to the clustering and colocalization of E-cadherin and episialin in the plasma membrane which caused a sterical hindrance of E-cadherin by episialin. Antibodies against episialin restored the E-cadherin function as these antibodies removed episialin from the clustering (9). The reorganization and movement of specific membrane proteins were associated with membrane microdomains $(3,21)$, enriched in glycosphingolipids, mediating cell-cell adhesion and modulating signal transduction (5).

In the present study we examined whether the ET-18OMe-induced effect on cell-cell adhesion in MCF-7/AZ breast cancer cells involved membrane microdomains. Our results showed that E-cadherin and episialin were associated with membrane microdomains in the two cell lines, which is in line with previous observations (17). The ET-18-OMe treatment led to an increased inclusion of the two membrane proteins in these domains in MCF-7/AZ cells. Simultaneously, lower levels were found in soluble fractions, 10-12, of the $\mathrm{MCF}-7 / \mathrm{AZ}$ cells, whereas no differences were observed in the MCF-7/6 cells. Since ET-18-OMe treatment did not alter the expression levels of E-cadherin and episialin (9), these findings suggest that ET-18-OMe induces translocation of Ecadherin and episialin to the membrane microdomains in MCF-7/AZ cells. Such translocation was reported for Fas/ CD95 in human leukemic cells (7).

In previous studies we reported the GSL composition of MCF-7/AZ and MCF-7/6 cells and demonstrated that ET-18OMe treatment had no influence on the expression levels $(16,17)$. Then, we examined changes in the cellular localization of E-cadherin and episialin and the possible connection with glycosphingolipids. Confocal microscopy revealed that ET-18OMe treatment resulted in the translocation of episialin from the apical to the lateral side and clustering and colocalization with E-cadherin, which is consistent with our previous study 

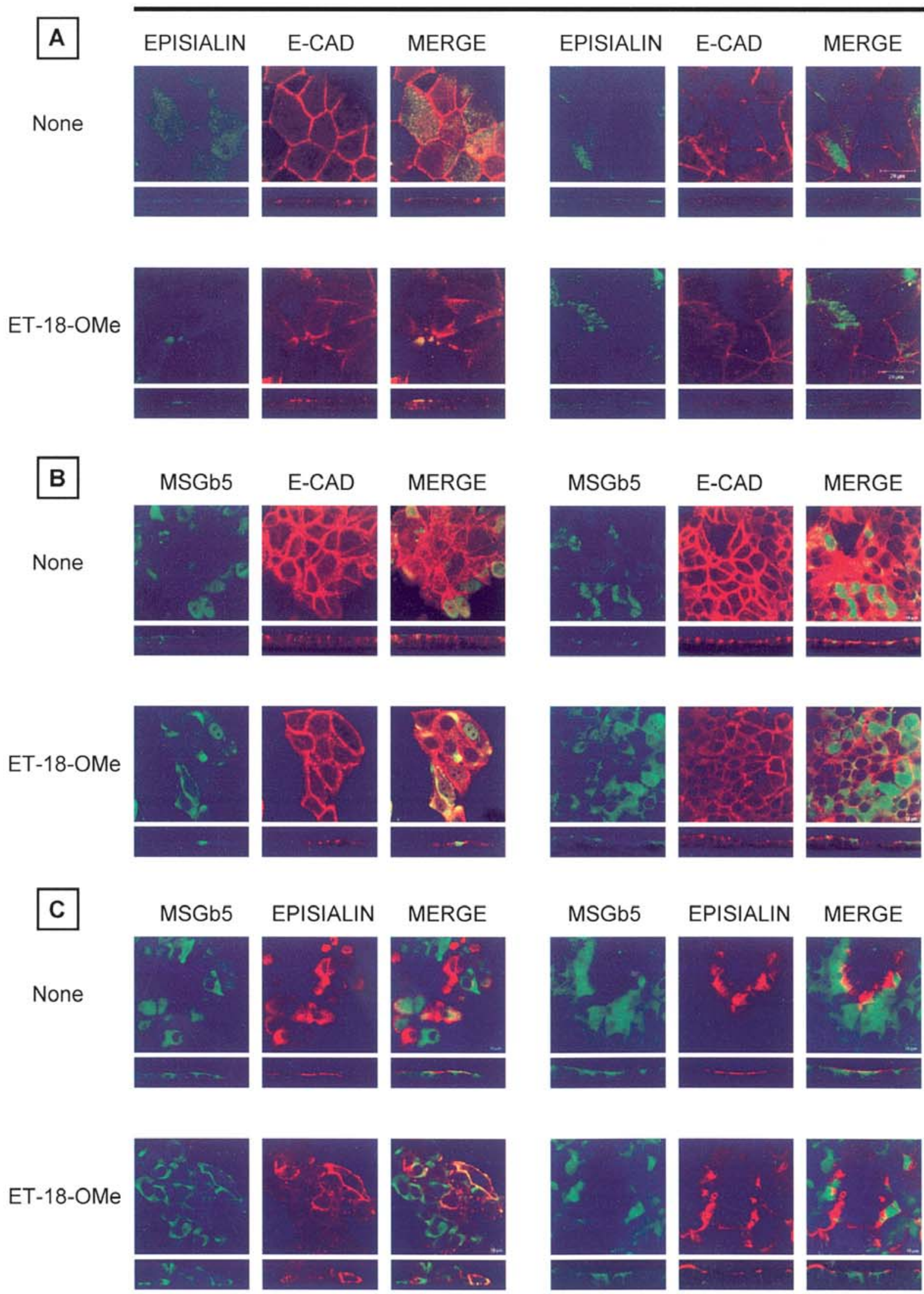

Figure 2. Localization and distribution patterns of E-cadherin, episialin and MSGb5 in the MCF-7/AZ and MCF-7/6 cells. The two cell lines were grown on glass coverslips, untreated or treated for $24 \mathrm{~h}$ with ET-18-OMe and further analyzed with the respective antibodies as described in Materials and methods. (A) Staining pattern of E-cadherin (E-CAD) and episialin, (B) MSGb5 and E-cadherin (E-CAD) and (C) MSGb5 and episialin in MCF-7/AZ (left panel) as compared to MCF-7/6 cells (right panel). Control stainings were performed without a primary antibody to measure possible cross-reaction or non-specific binding. All controls gave negative results (not shown). 

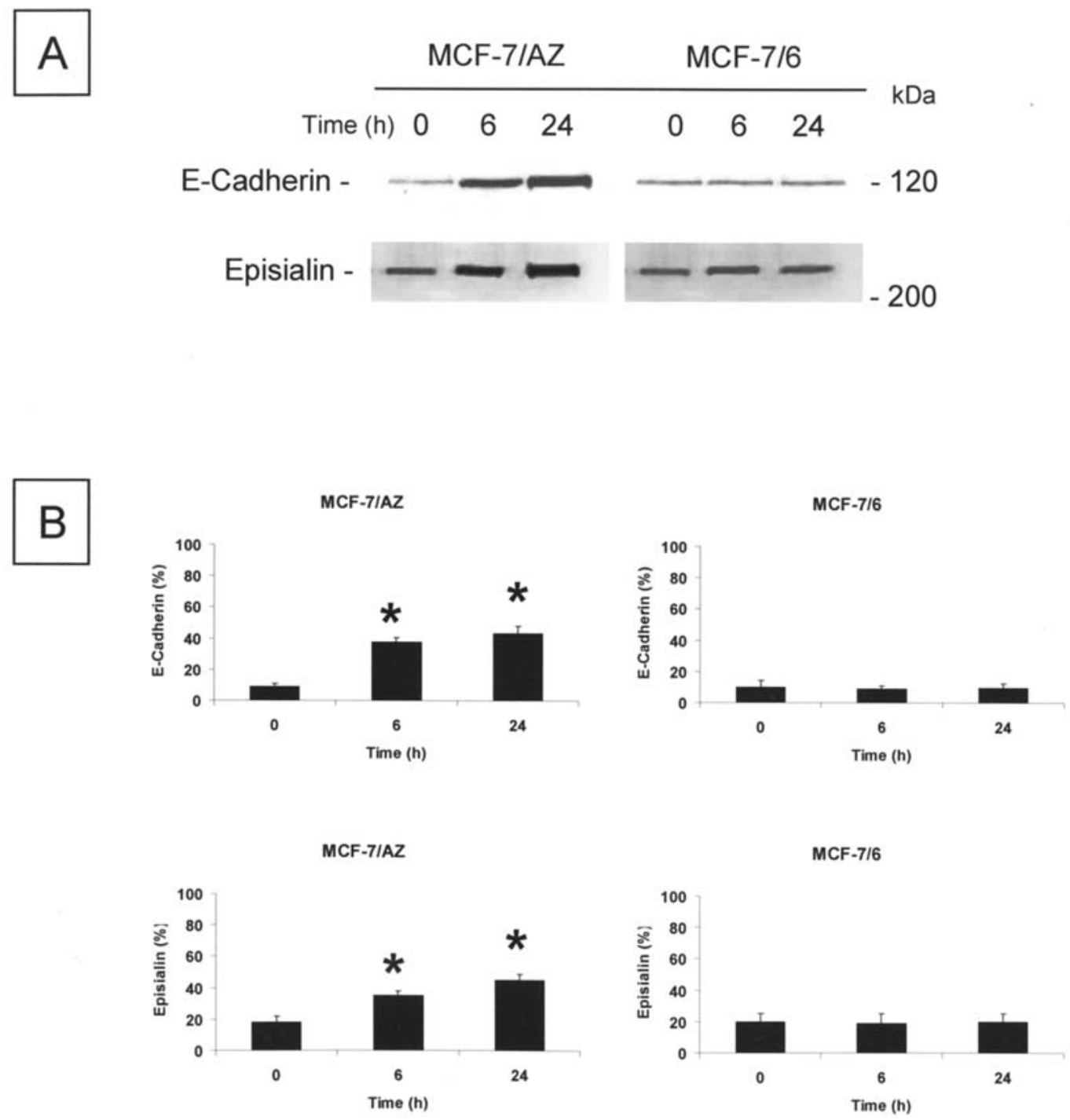

Figure 3. Association of MSGb5 with E-cadherin and episialin in the MCF-7/AZ and MCF-7/6 cells. Association of MSGb5 with E-cadherin and episialin in MCF-7/AZ (left panel) and MCF7/6 cells (right panel) upon treatment for the indicated times. MSGb5 was immunoprecipitated with IgG3 MSGb5 antibody. Aliquots were immunoblotted with anti-E-cadherin and anti-episialin. Lower panel, quantification by Scion image program. Bars indicate standard deviation; *statistical difference from untreated cells $(\mathrm{p}<0.05)$.

(9). In addition, ET-18-OMe caused clustering and translocation of the glycosphingolipid, MSGb5, to the lateral surface and colocalization with E-cadherin and episialin. We could exclude the involvement of several other GSLs present, since no significant changes in staining patterns were observed for the two cell lines. The obtained data indicated a close connection of E-cadherin and episialin with MSGb5 which was further confirmed by immunoprecipitation experiments, demonstrating the enhanced association of both E-cadherin and episialin with MSGb5 after ET-18-OMe treatment in MCF-7/AZ but not in MCF-7/6 cells. These results are in line with an increased inclusion of E-cadherin and episialin in membrane microdomain fractions in MCF-7/AZ cells (Fig. 1). Furthermore, a recent report demonstrated that the association of cadherin complexes with membrane microdomains is required for their adhesive function (22), which is in agreement with our previous study, where we showed that the cadherin complex remained intact upon ET-18-OMe treatment (9). The latter, together with the present findings support that an ET-18-OMe-induced loss of cell-cell adhesion and stimulation of invasion are due to sterical hindrance of functional E-cadherin by episialin, through translocation, clustering and colocalization of E-cadherin and episialin with MSGb5 into MSGb5-enriched membrane microdomains. Few studies exist on MSGb5, however, all of them link MSGb5 to invasion and metastasis $(16,17,23,24)$. Previous work showed that the stimulation of MSGb5, by its monoclonal antibody RM1, resulted in the clustering of MSGb5 with the subsequent activation of associated signaling molecules (17) leading to the enhanced secretion and activity of matrix metalloproteinases, which are known to stimulate invasion (16).

In conclusion, the present study showed that ET-18-OMe alters cellular adhesion, through the translocation and reorganization of crucial molecules in membrane microdomains. It also highlights that the differential composition of membrane microdomains is important for understanding basic cellular activities and mechanisms. 


\section{Acknowledgements}

This work was supported by the US National Institutes of Health (RR-16480) under the BRIN/INBRE program of the National Center for Research Resources and New Mexico Tech Startup Funds.

\section{References}

1. Pike LJ: Rafts defined: a report on the Keystone symposium on lipid rafts and cell function. J Lipid Res 47: 1597-1598, 2006.

2. Brown DA and London E: Structure and function of sphingolipidand cholesterol-rich membrane rafts. J Biol Chem 275: 17221-17224, 2000.

3. Simons $\mathrm{K}$ and Toomre D: Lipid rafts and signal transduction. Nat Rev Mol Cell Biol 1: 31-39, 2000.

4. Hakomori S, Handa K, Iwabuchi K, Yamamura S and Prinetti A: New insights in glycosphingolipid function: 'glycosignaling domain,' a cell surface assembly of glycosphingolipids with signal transducer molecules, involved in cell adhesion coupled with signaling. Glycobiology 8: 11-19, 1998.

5. Hakomori S: Tumor malignancy defined by aberrant glycosylation and sphingo (glyco) lipid metabolism. Cancer Res 56: 5309-5318, 1996.

6. Arthur G, Samadder P and Bittman R: ET-18-OCH3 inhibits the phosphorylation and activation of p70 S6 kinase in MCF-7 cells. Anticancer Res 25: 95-100, 2005.

7. Gajate $\mathrm{C}$ and Mollinedo F: The antitumor ether lipid ET-18$\mathrm{OCH}(3)$ induces apoptosis through translocation and capping of Fas/CD95 into membrane rafts in human leukemic cells. Blood 98: 3860-3863, 2001.

8. Mollinedo F, Gajate C, Martin-Santamaria S and Gago F: ET-18OCH3 (edelfosine): a selective antitumour lipid targeting apoptosis through intracellular activation of Fas/CD95 death receptor. Curr Med Chem 11: 3163-3184, 2004.

9. Steelant WF, Goeman JL, Philippe J, Oomen LC, Hilkens J, Krzewinski-Recchi MA, Huet G, Van der Eycken J, Delannoy P, Bruyneel EA and Mareel MM: Alkyl-lysophospholipid 1-Ooctadecyl-2-O-methyl-3-glycerophosphocholine induces invasion through episialin-mediated neutralization of E-cadherin in human mammary MCF-7 cells in vitro. Int J Cancer 92: 527-536, 2001.

10. Bracke ME, Van Larebeke N, Vyncke B and Mareel MM: Retinoic acid modulates both invasion and plasma membrane ruffling of MCF-7 human mammary carcinoma cells in vitro. $\mathrm{Br}$ J Cancer 63: 867-872, 1991.

11. Romijn JC, Verkoelen CF and Schroeder FH: Application of the MTT assay to human prostate cancer cell lines in vitro: establishment of test conditions and assessment of hormonestimulated growth and drug-induced cytostatic and cytotoxic effects. Prostate 12: 99-110, 1988.
12. Kannagi $\mathrm{R}$ and Hakomori $\mathrm{S}$ : A guide to monoclonal antibodies directed to glycotopes. Adv Exp Med Biol 491: 587-630, 2001.

13. Saito S, Levery SB, Salyan MEK, Goldberg RI and Hakomori S: Common tetrasaccharide epitope NeuAc $\alpha 2->3$ Gal $\beta 1->3$ (Neu-Ac $\alpha$ 2->6)GalNAc, presented by different carrier glycosylceramides or O-linked peptides, is recognized by different antibodies and ligands having distinct specificities. J Biol Chem 269: 5644-5652, 1994.

14. Yamamura $S$, Handa $K$ and Hakomori S: A close association of GM3 with c-Src and Rho in GM3-enriched microdomains at the B16 melanoma cell surface membrane: a preliminary note. Biochem Biophys Res Commun 236: 218-222, 1997.

15. Iwabuchi K, Yamamura S, Prinetti A, Handa K and Hakomori S: GM3-enriched microdomain involved in cell adhesion and signal transduction through carbohydrate-carbohydrate interaction in mouse melanoma B16 cells. J Biol Chem 273: 9130-9138, 1998.

16. Van Slambrouck S and Steelant WF: Clustering of monosialylGb5 initiates downstream signaling events leading to invasion of MCF-7 breast cancer cells. Biochem J 401: 689-699, 2007.

17. Steelant WF, Kawakami Y, Ito A, Handa AK, Bruyneel EA, Mareel MM and Hakomori S: Monosialyl-Gb5 organized with cSrc and FAK in GEM of human breast carcinoma MCF-7 cells defines their invasive properties. FEBS Lett 531: 93-98, 2002.

18. $\mathrm{Na} \mathrm{HK}$, Inoue $\mathrm{H}$ and Surh YJ: ET-18-O-CH3-induced apoptosis is causally linked to COX-2 up-regulation in H-ras transformed human breast epithelial cells. FEBS Lett 579: 6279-6287, 2005.

19. Dimanche-Boitrel MT, Meurette O, Rebillard A and Lacour S: Role of early plasma membrane events in chemotherapyinduced cell death. Drug Resist Update 8: 5-14, 2005.

20. Harrington KJ and Syrigos KN: The role of E-cadherin-catenin complex: more than an intercellular glue? Ann Surg Oncol 7: 783-788, 2001.

21. Jacobson K, Sheets ED and Simson R: Revisiting the fluid mosaic model of membranes. Science 268: 1441-1442, 1995.

22. Causeret M, Taulet N, Comunale F, Favard C and Gauthier-Rouviere C: $\mathrm{N}$-cadherin association with lipid rafts regulates its dynamic assembly at cell-cell junctions in C2C12 myoblasts. Mol Cell Biol 16: 2168-2180, 2005.

23. Saito S, Orikasa S, Ohyama C, Satoh M and Fukushi Y: Changes in glycolipids in human renal-cell carcinoma and their clinical significance. Int J Cancer 49: 329-334, 1991.

24. Saito S, Orikasa S, Satoh M, Ohyama C, Ito A and Takahashi T: Expression of globo-series gangliosides in human renal cell carcinoma. Jpn J Cancer Res 88: 652-659, 1997. 